ORIGINAL RESEARCH ARTICLE

\title{
Obstetric Fistula: A Narrative Review of the Literature on Preventive Interventions in sub-Saharan Africa
}

\author{
Eniya K. Lufumpa ${ }^{1}$ and Sarah Steele ${ }^{2}$ \\ Public Health, Epidemiology and Biostatistics, Institute of Applied Health Research, University of Birmingham, UK ${ }^{1}$; Global \\ Health and Policy Unit, Centre for Primary Care and Public Health, Barts and The London School of Medicine and Dentistry, \\ Queen Mary University of London, $\mathrm{UK}^{2}$
}

*For Correspondence: Email: ek1559@ bham.ac.uk; Phone: +44 (0)7481760244

\begin{abstract}
Obstetric fistulas are childbearing injuries that present a major public health issue, especially in the developing context. This study brings together the literature on the causes of these injuries in sub-Saharan Africa, as well as suggested interventions aimed at its prevention. Furthermore, it also aims to identify gaps in the research that need to be addressed. A systematic search of PubMed, Embase, Web of Science, and Scopus identified that extensive research has been carried out on the factors causing this injury, the main factors being complications during labour, three phases of delay, and culture. Nonetheless, there remains a dearth of literature detailing preventive measures. While few studies discussed preventive interventions that had been implemented, the literature does well to suggested preventive interventions. Suggested preventive measures target the community, as well as healthcare facilities. The literature also highlights the need for increased governmental support, as a means of preventing the development of fistulas. Using narrative review methods, we identify that the research focus remains primarily on the causes of obstetric fistula and ways of managing the injury, while paying less attention to means of preventing this injury. We therefore recommend further exploration of preventive measures. (Afr J Reprod Health 2016 (Special Edition); 20 [3]: 118-126).
\end{abstract}

Keywords: Obstetric fistula, Prevention, Intervention, Sub-Saharan Africa, Maternal health

\section{Résumé}

Les fistules obstétricales sont des blessures d'accouchement qui présentent un problème considérable de santé publique, en particulier dans le contexte des pays en voie de développement. Cette étude rassemble la documentation sur les causes de ces blessures en Afrique subsaharienne, ainsi que les interventions proposées visant à sa prévention. En outre, il vise également à identifier les lacunes dans la recherche qui doivent être abordées. Une recherche systématique sur les bases de données PubMed, Embase, Web of Science, et Scopus a révélé que des recherches approfondies ont été menées sur les causes de cette blessure, les principaux facteurs étant les complications pendant l'accouchement, les trois étapes de retard, et la culture. Néanmoins, il reste encore un manque de documentation détaillant les mesures préventives. Malgré que peu d'études aient discuté des interventions préventives qui avaient été mises en œuvre, la documentation a bienfait de proposer des interventions préventives. Ces mesures proposées ciblent la communauté, ainsi que les centres médicaux. La documentation met également en évidence la nécessité d'un soutien accru de la part du gouvernement, comme un moyen d'empêcher le développement des fistules. En utilisant des méthodes de recherche pour les examens narratifs, nous identifions que la documentation se concentre principalement sur les causes de la fistule obstétricale et les moyens de traiter cette blessure, tout en accordant moins d'attention à la prévention de cette blessure. Par conséquent, nous recommandons une exploration plus approfondi sur les mesures de prévention. (Afr J Reprod Health 2016 (Edition Spéciale); 20[3]: 118-126).

Mots clés: la fistule obstétricale, prévention, intervention, l'Afrique subsaharienne, la santé maternelle

\section{Introduction}

Obstetric fistula-a current global health concern, particularly for women in rural and developing settings - continues to receive extensive international attention ${ }^{1-5}$. The World Health Organization (WHO) estimates that over two million women live with untreated obstetric fistula in Asia and sub-Saharan Africa ${ }^{6}$. In sub-Saharan Africa - the region with the highest concentration of fistula patients globally-there is a belt extending from West to East Africa through the Horn of Africa where the condition is most prevalent ${ }^{7}$. Obstetric fistulas involve a hole or tear between the vagina and the bladder (known as vesico-vaginal fistula) and/or between the vagina and the rectum (known as recto-vaginal fistula) ${ }^{8}$. The outcome of such an injury is the incontinence of urine, blood, and/ or faeces, and, in the most severe cases, it can lead to maternal and fetal mortality ${ }^{9}$. The causes of obstetric fistulas are numerous, and include prolonged or obstructed 


\section{Lufumpa \& Steele}

labour, spontaneous abortion, and female genital mutilation, amongst other causes ${ }^{10}$.

However, the results of obstetric fistulas are not just physiological. Incontinence often results in women experiencing extensive social stigma and related mental health issues, with these women either marginalised by households and communities, or marginalising themselves ${ }^{1,5,11-13}$. Such isolation and stigma acts to decrease their chances of seeking treatment. Living outside the centre of their societies, these women are less likely to be aware that fistulas can be repaired and where to seek treatment ${ }^{13}$.

In light of such impacts, it is imperative to better understand and respond to obstetric fistulas, improving research into the causes, effective preventative interventions, and treatment of this condition. Accordingly, this study asks: What does the existing literature tell us about the factors that propagate the occurrence of obstetric fistulas? What interventions have been put in place to prevent the occurrence of it and how effective or ineffective have they been? What gaps remain in our knowledge of the development of fistulas that need to be filled in order to develop effective preventive interventions? In answering these questions, we adopt a systematic narrative review method that orients around the Sustainable Development Goals 3 and 5, which call for improvements in maternal health outcomes and the empowerment of women and the girl child. This study also aligns itself with other human rights declarations, which hold a minimum standard of maternal care prior to, during, and after giving birth, and thus any failure to provide such care is an injustice demanding an urgent response $\mathrm{e}^{3,12,14-16}$.

\section{Methods}

\section{Literature search}

The databases PubMed, Embase, Web of Science, and Scopus - four databases that provide the most up-to-date and relevant articles on life science and biomedical topics, including various public health issues - were searched with the terms ["obstetric fistula" AND "Africa" AND ("management" OR "policies" OR "protocol” OR “intervention") AND "prevention"] and were not limited to a specific

\section{Obstetric Fistula in Sub-Saharan Africa}

language. This search yielded results on only two databases. Consequently, we broadened the search terms to ["obstetric fistula" AND "Africa" AND "prevention"]. This search yielded 128 articles across all four databases. After removing duplicates, 79 articles remained (see Figure1).

\section{Article selection}

Articles were included in the study if they were: (1) written and published between 1990 and 2014; (2) cover the prevention of obstetric fistula in subSaharan Africa; and (3) written in English or French (the languages of the primary researcher). After reading through the 79 abstracts, 46 articles were excluded and 33 relevant articles remained (See Figure 1). It should be noted that three of the included studies did not provide significant relevant information and were not included in this study.

In addition to the search, the 33 articles' reference lists were examined to identify further studies on preventing obstetric fistulas in the region. This acted to crosscheck the data for any gaps, but no additional articles were identified that met the inclusion criteria.

\section{Data extraction and synthesis}

Similar to a systematic narrative review approach, we undertook a close reading of the abstract, article, and selected references, keeping careful note of the themes and meta-narrative ${ }^{17}$ around (1) the causes of obstetric fistula; (2) the extent to which it is deemed a problem in the sub-Saharan Africa; (3) methods to prevent obstetric fistula; and (4) interventions and treatments around those with obstetric fistula. We noted medical data, as well as accounts, country examples, case studies, and anecdotes.

This approach allowed us to undertake explanatory analysis, as well as to explore gaps in the current literature. By identifying core components of the narrative, especially around the types of processes used when transferring knowledge about prevention into policy and action, we were able to explore how the current research is both productive, but also limited ${ }^{18}$. We explore five core themes that emerged from close reading. 
Figure 1: Flow Diagram of the Article Selection Process

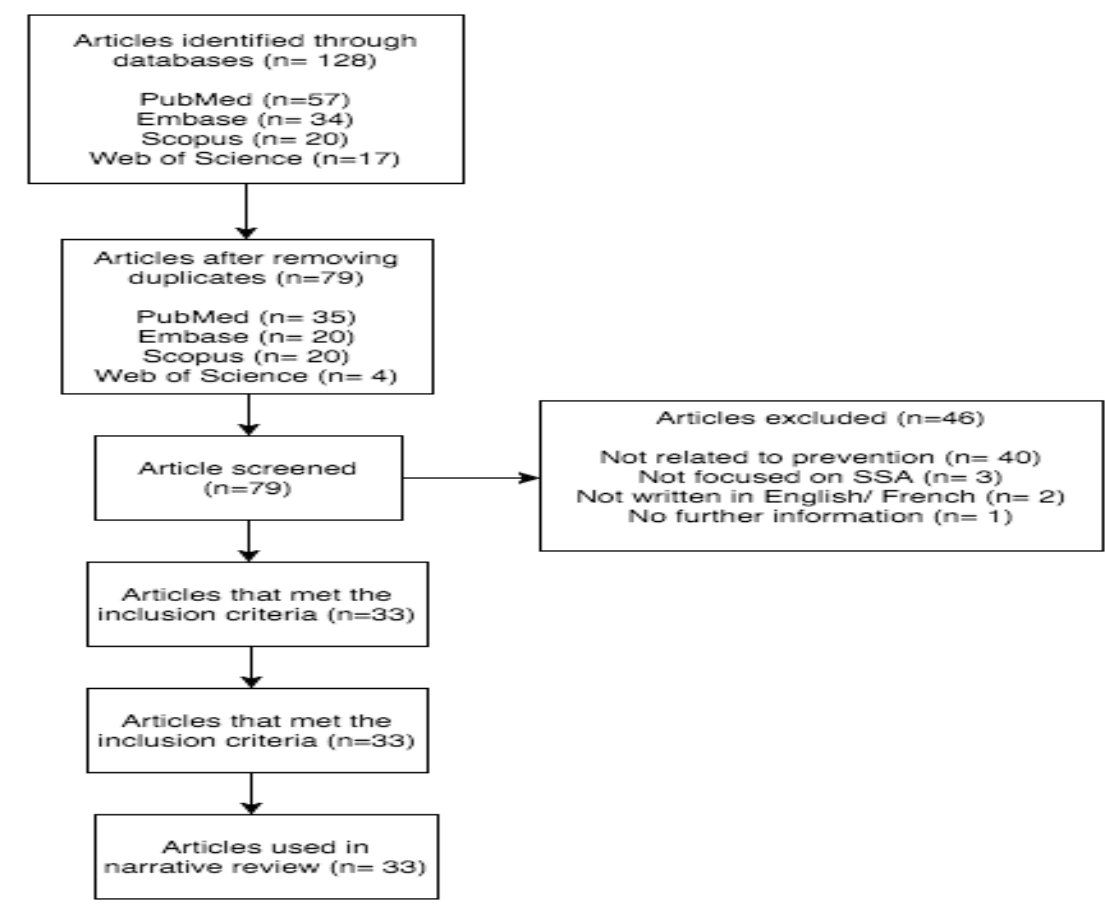

\section{Results}

The core themes identified included: (1) complications during labour; (2) three phases of delay; (3) the role of culture; (4) prevention; (5) the role of governments. We consider the data on each theme in turn.

\section{Complications during labour}

\section{Obstructed labour}

All 33 articles detailed the role of obstructed labour, which occurs when the progression of the fetus through the pelvis is stopped despite ongoing uterine contractions, as a cause of obstetric fistula ${ }^{19}$. As referenced in two articles, obstructed labour was said to arise due to various factors including but not limited to abnormal fetal position, the size of the fetus, and a narrow birthing canal ${ }^{20-21}$.

In considering regional issues around obstructed labour, only one article detailed that obstructed labour is common in African women due to the narrow build of their pelvis ${ }^{20}$. It was noted in eighteen articles that many African girls get married at an early age, which increases their risk for obstructed labour, which if not managed leads to prolonged labour ${ }^{1-4,11,12,14-15,19-28}$.

\section{Prolonged labour}

Six articles associated the development of fistulas with prolonged labour ${ }^{2,12,13,19,21,23}$, which they defined as the prolonged presence and pressure of the fetus against the soft tissues that separate the vagina from the bladder and/or the rectum, leading to their degeneration ${ }^{26}$. Crucially, the articles noted common divergence in understandings of the length of labour. One article relied on the WHO's definition of prolonged labour as labour lasting over 24 hours, while noting that in rural settings there are misconceptions as to the 'normal' length of labour ${ }^{21}$. Six articles detailed that standards set by the WHO are well understood by skilled birth attendants, however, most women in rural settings either deliver by themselves, in the company of family members, or with the aid of traditional birth assistants and are not aware that urgent obstetric care is needed past that point ${ }^{1,3,11,14,19,21}$. One study showed that in some settings, prolonged labour was not considered of enough importance to seek skilled medical care until two to five days had passed $^{21}$. Such understandings were connected to 


\section{Lufumpa \& Steele}

the concept of delay.

\section{Three phases of delay}

In 15 of the articles, a core focus was on the seminal work by Thaddeus and Maine on the Three Phases of Delay ${ }^{1-5,11,15-16,19-21,23-25,28}$, which is a conceptual model that identifies obstacles to the provision and timely utilisation of high quality obstetric care ${ }^{29}$. Consideration of the three phases occurred across these 15 articles $^{1-5,11,15-16,19-21,23-}$ 25,28 .

\section{Phase 1: delay in deciding to seek care}

The articles suggested that the factors greatly affecting the decision to seek care were various, including: distance, cost, sociocultural beliefs, and the perceived quality of care $e^{3-5,11,13-14,19,23-24,28}$. Importantly, one article emphasised that research has shown that an increase in the availability of services does not always increase the use of the services, leaving the issue of delay in decision to seek care an important focus for researchers ${ }^{29}$.

The core focuses of the articles remained on two central factors driving Phase 1 delay: geography and cost. Eight of the articles mention that, more often than not, in rural areas health care facilities are sparse, resulting in people having to travel great distances to access health care ch-5,11,15,24- $^{3}$ ${ }^{28}$. Such geographical distance not only was connected to travel time (impacting on Phase 2 delay), but connected to the idea of cost. One study attributed delay to the financial cost of treatment at short distance ${ }^{29}$, while seven other articles emphasised the role of financial costs ${ }^{4-}$ $5,11,14,21,23,25$, which included: the cost of care at a facility, transportation costs, and the cost of upkeep for the patient and accompanying family. Five studies confirmed a more multi-faceted notion of financial constraints ${ }^{4,11,20,23,26}$, while two others considered the issue of opportunity costs as a great concern, alongside upkeep for the patient and accompanying family ${ }^{19,28}$. The role of time was also considered, with a suggestion that many women would rather spend time doing more productive things than seeking care ${ }^{28}$.

\section{Phase 2: delay in reaching a medical facility}

Even when the decision is made to seek professional medical care, 14 of the articles

\section{Obstetric Fistula in Sub-Saharan Africa}

highlighted a lack of access to transportation as a core factor in delay ${ }^{2-4,11,15,19-20,22-24,26-28}$. Five studies pointed out the uncertainty concerning the actual accessibility of facilities, due to the often-uneven distribution of services ${ }^{11,19,27-29}$.

The idea of geographical distribution leading to Phase 2 delay played out in four articles $^{4,20-21,28}$. Two articles emphasised that in developing countries there has always been a shortage of healthcare facilities, particularly in rural areas, as the vast majority of healthcare facilities are located in urban areas ${ }^{21,28}$. Two further articles suggested distance is often compounded by the terrain, with rural areas having unpaved and dilapidated roads, which are rough conditions to travel ${ }^{4,20}$. Such factors explained the higher rates of obstetric fistula in rural locations.

\section{Phase 3: delay in receiving adequate care}

Core to the idea of Phase 3 is the notion of delay that falls within the jurisdiction of elites, including government officials, hospital administrators, physicians, midwives, and their professional organizations ${ }^{19}$. Such delay is conceived in a multifaceted manner, considering health systems factors, as well as facility-based and professional failures. Five articles attribute this delay to a lack of the necessary equipment, drugs, and a shortage of skilled staff $\mathrm{f}^{3-4,11,23-24}$. Six articles point out that some patients are able to reach the facility in a timely manner; however, due to the inadequacy of the facility to meet their needs, they are referred to other facilities, which further delays appropriate treatment ${ }^{1-3,13,15,23}$.

A core narrative around Phase 3, however, is medical error or incompetency. Four articles referenced the occurrences of deaths and fistulas due to unskilled decisions, and the neglect of patients $^{2,4,25,28}$. Seven articles suggested that the failures of health services lie in the fact that facilities are often ill-staffed and illequipped ${ }^{1,4,15,18,24-26}$. Thus, it is not just the number of staff that are available that impacts of the availability of adequate care, but also a question of professional competence. One article connects this to resources being mismanaged ${ }^{29}$. Another study highlighted how such failures lead people to opt for traditional methods of managing obstetric complications ${ }^{4}$. 


\section{Lufumpa \& Steele}

\section{The role of culture}

Culture played a central role in ten of the articles, with particular emphasis placed on how culture has a direct impact on what type of management for obstetric complications is sought ${ }^{3-4,11,13,16,19,23-}$ 24,27-28. Notably, the articles emphasised that culture plays a significant role in shaping views within a society ${ }^{19}$, and one article noted that culture influenced lives much more significantly in individuals without formal education ${ }^{24}$. Again, geography was emphasised, with those in rural settings observed to hold cultural beliefs that seem to be more anecdotal and lack reliable evidence as to how effective they are ${ }^{19}$. Notably, culture was said to impact the beliefs about obstetric fistula, perceptions of hospitals, and on sociocultural practices that increase risk.

\section{Beliefs as to why obstetric fistulas develop and their treatments}

Seven articles emphasised the role that cultural beliefs have in attributing obstetric fistula to nonobstetric related mechanisms $s^{4,11,16,19,23,27,28}$. Six articles recounted the general belief in rural African communities that obstetric complications are a result of factors such as the will of God, heredity, and witchcraft ${ }^{4-5,19,21,24,28}$. Crucially, the articles emphasised that these societies are heavily based on tradition and spirituality, leading a physiological problem to be interpreted as caused by displeasing the customs of that society ${ }^{21}$. Such beliefs suggest that when obstetric complications arise, the women themselves are often to blame ${ }^{19}$. Five articles suggest that before women are able to seek medical attention, in some societies she is required to confess and apologize for her transgressions and then performs a series of rituals involving her husband ${ }^{4-5,14,24,28}$. Such beliefs are connected to Phase 1 and 2 delays. Six articles point to the result on such cultural beliefs being the perception that the solution to fistula lies in religious figures ${ }^{3-4,13,16,19,24}$. Such beliefs deem prayers and religious rituals as a means of treating these injuries, but do nothing to directly aid the progression of labour ${ }^{19}$. Two articles reveal that in some cultures, women who give birth with little assistance are more respected than those who require clinical intervention ${ }^{4,28}$. Two studies reveal

\section{Obstetric Fistula in Sub-Saharan Africa}

that attitudes such as these, coupled with the fear of hospitals, lead women to delay or to fail to seek timely medical intervention ${ }^{4,28}$.

\section{Perception of Hospitals}

These two studies detailed specifically that fear of healthcare facilities shaped responses to both labour and the condition itself $f^{4,28}$. One study detailed how hospitals were perceived as places where people go to $\mathrm{die}^{13}$.

Two studies also detailed how perceptions of the quality of care affect when, and where women seek care ${ }^{13,28}$. Such perceptions were irrespective of data, which notably itself is conflicted, with some suggesting such negative perceptions are not always justified (as some poor delivery outcomes are the result of late admission to hospital) ${ }^{13}$, while seven articles agreed care may be substandard, justifying the communities' perceptions ${ }^{1,4,15,24-26,28}$.

\section{Cultural practices}

Cultural practices around marriage were emphasised as playing a role in the perpetuation of obstetric fistulas ${ }^{3-4,11,16,19-21,24,28}$. Practices such as parents marrying daughters in exchange for a bride price, as a means of ensuring their daughter's financial security, were emphasised as holding a role in one article ${ }^{16}$. In some cultures, dowries decrease with age, this serves as an incentive for parents to have their daughters married at a younger age. Consequently, newlywed girls are pressured to have children quickly as a means of proving their fertility and securing their status, and respect as an 'adult' ${ }^{16}$. Fourteen articles discussed child marriage as having a direct impact on fistula rates, which are highest in areas where women are married very young ${ }^{2-5,12,14,16,20-24,26,30}$. Six studies noted that people participating in child marriage practices are not fully cognizant of the negative health effects it has on the young $\operatorname{girl}^{2,5,12,16,21,24}$. The articles also drew on human rights discourses. Two studies referenced international human rights norms around marriageable age as 18, which is not properly implemented in all countries ${ }^{16,24}$. Eight articles referred to child marriage as a violation of human rights ${ }^{2-4,12,14,15,16,22}$. Five articles noted such practices were driven by excessive poverty, and 


\section{Lufumpa \& Steele}

thus had cultural and socio-economic sources $5,12,16,21,24$. Such diverse causes thus necessitated different responses, including different prevention methods.

\section{Suggested preventive measures}

Twenty-one of the articles proposed prevention methods, which included community-based, facility-based, and antenatal centred approaches.

\section{Community-based}

Thirteen articles promoted preventive action targeting the community with a view to addressing the low status of women ${ }^{1,3,5,11,14,16,19-21,24-28}$. Three studies also point out that community providers, religious leaders, and other people deemed respectable within the community hold a core role in what happens to pregnant women ${ }^{3,13,15}$, particularly in light of their capacity to perpetuate or oppose some of the cultural practices like child marriage that drive obstetric fistula ${ }^{3,16}$. Three studies detailed a role for fistula survivors to act as 'ambassadors' within their communities, detailing how they are able to share their stories and advise pregnant women to seek antenatal care and birthing with a trained birth attendant ${ }^{2,11,22}$. Three studies also advised the use of media, especially radio and social media, as a means of raising awareness ${ }^{3,13-14}$.

\section{Healthcare facilities}

Ten articles highlighted that the availability of skilled care during the prenatal period and during labour is central to prevention of fistulas ${ }^{2-}$ $5,11,13,15,19,27-28$. One study suggested providing current healthcare providers, even those that do not specialize in obstetrics, with a course on screening for women at risk of developing fistulas, so as to refer them to adequate facilities in a timely manner ${ }^{3}$. Emphasis was placed on the role of training and adequate care.

\section{Antenatal care}

Ten articles focused on antenatal care as key to helping identify women who are at risk for the formation of fistulas and thus prevent $\mathrm{it}^{3-4,11,15,20-}$ 24,27-28. Regular antenatal visits serve to educate

\section{Obstetric Fistula in Sub-Saharan Africa}

women on birth preparedness, and the importance of a trained birth attendant, as well as information about emergency obstetric care ${ }^{11}$.

Four studies examined the use of maternal waiting homes, which have been associated with a decrease in the need for emergency obstetric care ${ }^{3-}$ $4,24,28$. These are facilities within the proximity of a medical facility that is able to provide such care when it is needed ${ }^{3}$.Women who are identified as having 'high-risk' pregnancies are taken as they await their delivery, and can easily be taken to an adequate facility if the need arises ${ }^{3}$. Generally, these facilities also serve to address the roles that distance and transport costs play ${ }^{24}$.

\section{Government involvement}

Fifteen articles acknowledge that, in light of such care needs and systems developments, there is a need for an increased support at a governmental level, as there are countries where support is either inconsistent or weak ${ }^{4-5,11-12,14-16,19,21,23-25,27-28}$. One article focused on the use of policies addressing sexual and reproductive health ${ }^{11}$. Another study suggests that governments must tackle the issue of the 'brain drain', which involved subsidizing and maintaining national health services to a sufficient standard to encourage health professionals to stay within the country ${ }^{24}$. Another study proposed that governments must enforce the minimum marriage age, while ensuring that education is free to women and girls and that there are employment opportunities after graduation ${ }^{7}$. Such diverse accounts suggested a range of involvements for governments across healthcare systems and around the social determinants of health more broadly, discussed in the sections that follow.

\section{Discussion}

The 33 articles selected for review accounted for a range of biomedical and socio-cultural issues around obstetric fistula, indicating diverse potential for improved responses. The literature was heavily focused on the causes of fistula, rather than a discussion of preventive strategies. The majority of preventive strategies discussed in the articles were in the form of suggestions, in lieu of evaluations of actual interventions that had been put in place. Thus, it is essential to research the 


\section{Lufumpa \& Steele}

effectiveness of preventive interventions implemented, as well as those implemented in the future, rather than suggested preventive measures.

The literature on biomedical factors suggested that they lead directly to the formation of fistulas, with particular focus placed on prolonged labour and obstructed labour ${ }^{12,14-15}$. Attributed in part to inadequacies of the health systems to provide adequate care to address obstetric complications, there is a clear need to conduct further research on facilities and healthcare provision. Indeed, observations that obstetric fistulas can be due to limited budgets and a different order of priorities ${ }^{15}$, suggest the need for a health systems and policy lens in future research.

Observations around infrastructure, the lack of specialized health professionals, and the lack of equipment ${ }^{19}$, demonstrate that policy level decisions must be locally responsive, with hospital level audits also conducted to drive appropriate resource decisions. The prioritization of women's and girls' health should also take place with adequate focus on obstetric emergencies ${ }^{4,15,19}$. However, such observations were often made anecdotally, and thus more research needs to be carried out in assessment of current health systems in the region and the factors that impede the prevention of this condition. Such research must consider not only policy and facilities roles, but also explore current and potential interface with the Third Sector.

Notably, the importance of the Three Phases of Delay model in 15 of the articles shows the considerable role that these phases play in the development of fistulas ${ }^{1-5,11,15-16,19,21-26}$. Crucially, the research does not indicate whether all the phases of delay play an equal role in the development of fistulas. One article notably points out that phase two and phase three delays appear to be the most amenable to solution ${ }^{25}$. Greater research and surveillance of delays is needed to inform how preventive strategies can be built. Additionally, the current research focuses greatly on obstetric fistulas that are acquired as a result of obstetric complications within the demographic of women who are married. Further research should endeavour to examine obstetric fistulas in the context of violence against women, including

\section{Obstetric Fistula in Sub-Saharan Africa}

women who have experienced sexual trauma, female genital mutilation, or surgical errors, amongst others.

Importantly, such research and obstetric prioritization requires attending to the low status of women within societies where this injury is most prevalent; a shift that must be multi-level. The crucial account of the role culture plays in the decision to seek care requires education and status shifts, which require elite, community leadership, and public shifts. Within developing settings, women are seen as financial burdens on their parents and as such are married off at an early age: a harmful practice that reinforces the social understanding that value is placed in the ability to have children ${ }^{16}$. Addressing these factors requires not just broader social level changes, but also reframing the research on health systems to pay greater attention to the inequalities that must be addressed in the sub-Saharan area.

Crucially, the focus on the human rights aspects of fistula in the context of child marriage demonstrates the recognised connection obstetric fistula has with human rights and the Sustainable Development Goals. It is crucial to seize on momentum to improve accelerated access to sexual and reproductive health, as well as to protect women's rights. Virtually eliminated in the developed world, the prevalence of fistulas across sub-Saharan Africa emphasises a critical need for developing access, equity and equality. Alongside national and community level responses, international support for mechanisms for surveillance, monitoring and improving responses, and integration will play a crucial role. Few articles articulated these possibilities and thus focused human rights research within the context of global health and biomedicine are needed.

\section{Limitations of the Study}

Notably, that the articles were found in the four databases limits the research and thus it is possible such explorations are made in journals indexed elsewhere and open access materials of a non-peer reviewed nature. There is certainly scope to greatly expand this study in the future, as this will likely yield further, and more revealing results. Additionally, while obstetric fistulas are a historic issue, the focus on sub-Saharan Africa has only 


\section{Lufumpa \& Steele}

occurred within the last few decades, and thus various studies note that such research is significantly focused on hospital-based retrospective research at present ${ }^{5}$. This greatly limited the search, as there was not much research that reflects the work of what has been done in attempt to prevent this condition. Again, further expanded research is recommended.

\section{Conclusion}

Through the form of a systematic narrative review, this study examined the various factors that lead to the formation of fistulas, and any accounts of preventive interventions. The review revealed that literature on the injury pays less attention to the dimension of prevention, despite that, as the ageold adage goes, 'prevention is better than the cure'. A focus on prevention will entail a clear focus on the overall improvement of the lives of women and girls in the region.

Integrally, prevention requires the empowerment of women and girls as a precondition. Indeed, the 5th Sustainable Development Goal concerning the improvement of maternal health can one day be realized if broader goals around universal access to healthcare (especially reproductive healthcare), girls' education, eradication of gender-based violence, and gender equity are achieved ${ }^{27}$. The virtual eradication of this condition in developed contexts evidences the possibilities for eradication globally. This entirely preventable injury thus remains one of the clearest reminders of inequality, and thus demands immediate and dedicated attention by policy-makers, practitioners, stakeholders and communities alike.

\section{Contributions}

The research was carried out by Eniya Lufumpa, under the direct supervision of Dr Sarah Steele, who oversaw the research and contributed to all stages of production of the manuscript. All authors have approved the final version of the manuscript.

\section{References}

1. De Bernis, L. and Lewis, G. (2007). Obstetric fistula: guiding principles for clinical management and programme development, a new WHO guideline.

\section{Obstetric Fistula in Sub-Saharan Africa}

International Journal of Gynecology $1 \&$ Obstetrics, 99, pp.117--121.

2. Donnay, F. and Weil, L. (2004). Obstetric fistula: the international response. The Lancet, 363.

3. Miller, S., Lester, F., Webster, M. and Cowan, B. (2005). Obstetric fistula: a preventable tragedy. Journal of Midwifery \\& Women's Health, 50(4), pp.286--294.

4. Velez, A., Ramsey, K. and Tell, K. (2007). The Campaign to End Fistula: What have we learned? Findings of facility and community needs assessments. International Journal of Gynecology I\& Obstetrics, 99, pp.143--150.

5. Zheng, A. and Anderson, F. (2009). Obstetric fistula in low-income countries. International Journal of Gynecology \\& Obstetrics, 104(2), pp.85--89.

6. who.int, (2014). WHO 10 facts on obstetric fistula. [online] Available at: http://www.who.int/features /factfiles/obstetric_fistula/en/.

7. Ojanuga, D. (1991). Preventing birth injury among women in Africa: Case studies in Northern Nigeria. American journal of orthopsychiatry.

8. Bangser, M. (2006). Obstetric fistula and stigma. The Lancet, 367(9509), pp.535--536.

9. Cook, R., Dickens, B. and Syed, S. (2004). Obstetric fistula: the challenge to human rights. International Journal of Gynecology \\& Obstetrics, 87(1), pp.72-77.

10. Banke-Thomas, A., Kouraogo, S., Siribie, A., Taddese, H. and Mueller, J. (2013). Knowledge of Obstetric Fistula Prevention amongst Young Women in Urban and Rural Burkina Faso: A Cross-Sectional Study. PloS one, 8(12), p.85921.

11. Capes, T., Ascher-Walsh, C., Abdoulaye, I. and Brodman, M. (2011). Obstetric fistula in low and middle income countries. Mount Sinai Journal of Medicine: A Journal of Translational and Personalized Medicine, 78(3), pp.352--361.

12. Osotimehin, B. (2013). Obstetric fistula: ending the health and human rights tragedy. The Lancet, 381(9879), pp.1702--1703.

13. Wegner, M., Ruminjo, J., Sinclair, E., Pesso, L. and Mehta, M. (2007). Improving community knowledge of obstetric fistula prevention and treatment. International Journal of Gynecology $1 \&$ Obstetrics, 99, pp.108--111.

14. Baptiste, D., Kapungu, C., Khare, M., Lewis, Y. and Barlow-Mosha, L. (2010). Integrating women's human rights into global health research: an action framework. Journal of Women's Health, 19(11), pp.2091--2099.

15. Mselle, L., Moland, K., Mvungi, A., Evjen-Olsen, B. and Kohi, T. (2013). Why give birth in health facility? Users' and providers' accounts of poor quality of birth care in Tanzania. BMC health services research, 13(1), p.174.

16. Nour, N. (2006). Health consequences of child marriage in Africa. Emerging infectious diseases, 12(11), p.1644.

17. Greenhalgh, Trisha. How to Read a Paper: The Basics of 


\section{Lufumpa \& Steele}

Evidence-based Medicine. Chichester, West Sussex, UK: Wiley-Blackwell, 2010. Print.

18. Wilson, K., Brady, T. and Lesesne, C. (2011). An

Organizing Framework for Translation in Public Health: The Knowledge to Action Framework. Preventing Chronic Disease, 8(2, A46), pp.1-7.

19. Wall, L. (2012). Overcoming phase 1 delays: the critical component of obstetric fistula prevention programs in resource-poor countries. BMC pregnancy and childbirth, 12(1), p.68.

20. Tsui, A., Creanga, A. and Ahmed, S. (2007). The role of delayed childbearing in the prevention of obstetric fistulas. International Journal of Gynecology I\& Obstetrics, 99, pp.98--107.

21. Wall, L. (2006). Obstetric vesicovaginal fistula as an international public-health problem. The Lancet, 368(9542), pp.1201--1209.

22. Callister, L. (2013). Silent Suffering: The Tragedy of Obstetric Fistula. MCN: The American Journal of Maternal/Child Nursing, 38(3), p.187.

23. Keri, L., Kaye, D. and Sibylle, K. (2010). Referral practices and perceived barriers to timely obstetric care among Ugandan traditional birth attendants (TBA). African health sciences, 10(1), p.75.

\section{Obstetric Fistula in Sub-Saharan Africa}

24. Norman, A., Breen, M. and Richter, H. (2007).

Prevention of obstetric urogenital fistulae: some thoughts on a daunting task. International Urogynecology Journal, 18(5), pp.485--491.

25. Ramphal, S. and Moodley, J. (2006). Vesicovaginal fistula: obstetric causes. Current Opinion in Obstetrics and Gynecology, (18).

26. Shefren, J. (2009). The tragedy of obstetric fistula and strategies for prevention. American Journal of Obstetrics \& Gynecology.

27. Wall, L. (1996). Obstetric fistulas in Africa and the developing world: new efforts to solve an age-old problem. Women's Health issues, 6(4), pp.229--234.

28. Wall, L. (2012). Preventing obstetric fistulas in lowresource countries: insights from a Haddon matrix. Obstetrical $\backslash \&$ gynecological survey, 67(2), pp.111$-121$.

29. Thaddeus, S. and Maine, D. (1994). Too far to walk: maternal mortality in context. Social science $1 \&$ medicine, 38(8), pp.1091--1110.

30. Browning, Andrew, Jenifer E. Allsworth, and L. Lewis Wall. "The Relationship Between Female Genital Cutting and Obstetric Fistulas." Obstetrics and gynecology 115.3 (2010): 578-583. PMC. 